\title{
A CBR System: The Core of an Ambient Intelligence Health Care Application
}

\author{
Juan M. Corchado, Javier Bajo, and Yanira de Paz \\ Departamento Informática y Automática \\ Universidad de Salamanca \\ Plaza de la Merced s/n, 37008, Salamanca, Spain \\ corchado@usal.es, jbajope@usal.es , yanira@usal.es
}

\section{Introduction}

This paper presents a case-based reasoning system developed to generate an efficient and proactive ambient intelligent application. Ambient Intelligence (AmI) proposes a new way to interact between people and technology, where this last one is adapted to individuals and their context (Friedewald and Da Costa 2003). The objective of Ambient Intelligence is to develop intelligent and intuitive systems and interfaces capable to recognize and respond to the user's necessities in a ubiquitous way, providing capabilities for ubiquitous computation and communication, considering people in the centre of the development, and creating technologically complex environments in medical, domestic, academic, etc. fields (Susperregui et al. 2004). Ambient Intelligence requires new ways for developing intelligent and intuitive systems and interfaces, capable to recognize and respond to the user's necessities in a ubiquitous way, providing capabilities for ubiquitous computation and communication. The multiagent systems (Wooldridge and Jennings 1995) have become increasingly relevant for developing distributed and dynamic intelligent environments. A case-based reasoning system (Aamodt and Plaza 1994) has been embedded within a deliberative agent and allows it to respond to events, to take the initiative according to its goals, to communicate with other agents, to interact with users, and to make use of past experiences to find the best plans to achieve goals. The deliberative agent works with the concepts of Belief, Desire, Intention (BDI) (Bratman 1987), and has learning and adaptation capabilities, which facilitates its work in dynamic environment.

With the appearance of AmI-based systems, one of the most benefited segments of population will be the elderly and people with disabilities. It will improve important aspects of their life, especially health care (Emiliani and Stephanidis 2005). There is an ever growing need to supply constant care and support to the disabled and elderly (Nealon and Moreno 2003) and the drive to find more effective ways to provide such care has become a major challenge for the scientific community. Today, the number of Europeans over 60 years represents more than $25 \%$ of the population and it is estimated that in 20 years this percentage will rise to one third of the population (Camarinha-Matos and Afsarmanesh 2002). In the United States of America it is expected that in 2020 people over 60 will represent about 1 of 6 citizens (Kohn et al. 1999). Furthermore, over $20 \%$ of people over 85 years have a limited capacity for independent living, requiring continuous monitoring and daily care. The Institute of Medicine

B. Prasad (Ed.): Soft Computing Applications in Industry, STUDFUZZ 226, pp. 311-330, 2008. springerlink.com 
has studied the role of information technology in improving health care delivery in the US. In (Kohn et al. 1999), the Institute presents a strategy and an action plan to foster innovation and improve the delivery of care. The need to reinvest in the system is underlined and as such six health care aims are defined; to be safe, effective, patient-centered, timely, efficient and equitable. Ten guidelines for the redesign of the system are given attention on the role of the patient and improvements in knowledge, communication and safety mechanisms. Moreover, the Institute proposes a strategy to improve safety in health care based on the study of medical errors. The proposed system presented here has been conceived and developed taking these considerations into account.

The importance of developing new and more reliable ways to provide care and support to the elderly is underlined by this trend (Camarinha-Matos and Afsarmanesh 2002), and the creation of secure, unobtrusive and adaptable environments for monitoring and optimizing health care will become vital. Some authors (Nealon and Moreno 2003) consider that tomorrow health care institutions will be equipped with intelligent systems capable of interacting with humans. Multi-agent systems and architectures based on intelligence devices have recently been explored as supervision systems for medical care for the elderly or Alzheimer patients (Corchado et al. 2007). These intelligence systems aim to support them in all aspects of their daily life, predicting potential hazardous situations and delivering physical and cognitive support.

Radio Frequency IDentification (RFID) (Sokymat 2006) is an automated datacapture technology that can be used to electronically identify, track, and store information about products, items, components or people. It is most frequently used in industrial/manufacturing, transportation, distribution, and warehousing industries, however, there are other growth sectors including health care. The proposed system uses microchips mounted on bracelets worn on the patient's wrist or ankle, and sensors installed over protected zones, with an adjustable capture range up to 2 meters. The microchips or transponders help locate the patients, which can be ascertained by consulting the CBR agents installed in personnel PDAs.

The proposed system has been developed for facilitating the management and control of geriatric residences. The aim of this paper is to present the CBR based agent and to demonstrate how its planning mechanism improves the medical assistance in geriatric residences. These agents also facilitate the nurses' and doctors' work by providing updated information about patients and emergencies, as well as historical data. We believe that our system can easily be adapted to work in many other similar industrial environments.

This chapter is organized as follows: the next section describes the need for new Ambient Intelligence solutions and the advantages of using agents and case-based planning mechanisms for the development of intelligent environments. Section 3 describes the proposed CBP mechanism in detail. In Section 4 the mechanism proposed in Section 3 is applied to a geriatric residence. Finally, Section 5 presents the results and conclusions obtained in this research.

\section{CBR-Based Architecture for AmI Solutions}

Case-based Reasoning (CBR) is a type of reasoning based on the use of past experiences to solve new problems (Aamodt and Plaza 1994). The purpose of a CBR system 
is to solve new problems by adapting solutions that have been used to solve similar problems in the past. A case can be defined as a past experience, and is composed of three elements: A problem description which describes the initial problem, a solution which provides the sequence of actions carried out in order to solve the problem, and the final state which describes the state achieved once the solution was applied.

A CBR system manages cases (past experiences) to solve new problems. The way in which cases are managed is known as the CBR cycle. The CBR cycle shown in Figure 1 consists of four sequential phases: retrieve, reuse, revise and retain. The retrieve phase starts when a new problem description is received. Similarity algorithms are applied in order to retrieve from the cases memory the cases with a problem description more similar to the current one. Once the most similar cases have been retrieved, the reuse phase begins. In this phase the solutions of the cases retrieved are adapted to obtain the best solution for the current case. The revise phase consists of an expert revision of the solution proposed. Finally, the retain phase allows the system to learn from the experiences obtained in the three previous phases and updates the memory case in consequence.

AmI proposes new possibilities for solving a wide scope of problems and a new interaction way between people and technology, where this last one is adapted to individuals and their context, showing a vision where people are surrounded by intelligent interfaces merged in daily life objects (Emiliani and Stephanidis 2005). This

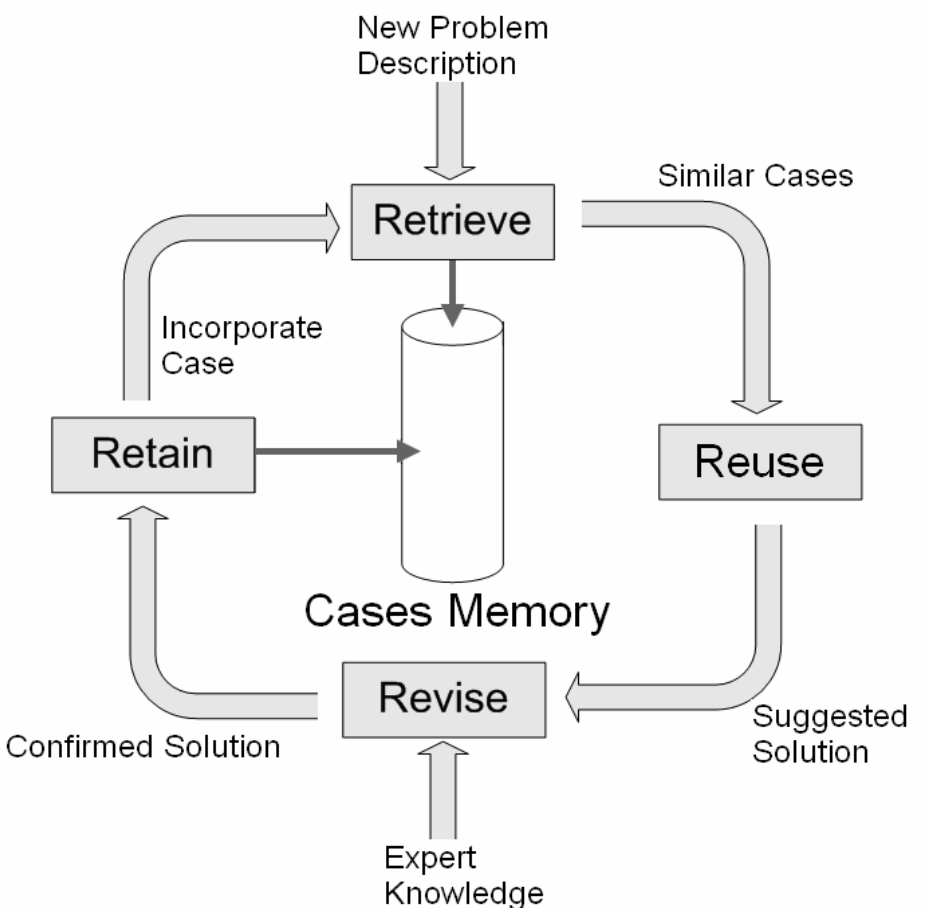

Fig. 1. Diagram including a CBR-BDI agent reasoning cycle 
adaptation requires creating computing-capable environments with intelligent processing and communication services. One of the possibilities for executing complex computational tasks is the use of CBR systems, which are capable of learning from their initial knowledge and autonomously adapting themselves to environmental changes. As CBR systems use their experiences from previous similar situations to resolve a new problem, it will be possible to personalize a user access if we take into account similar past cases. These similar past cases can contain memories about users with similar profiles trying to resolve a problem in similar context conditions.

The CBR adaptation and learning mechanism needs updated information from the environment and communication paths, with both the system users and the system elements. The multiagent technology (Wooldridge and Jennings 1995) can be used for solving distributed systems in which CBR systems may act. In this paper it is proposed that the use of agents reason and solve problems using embedded CBR systems (Corchado and Laza 2003). The agents and multi-agent systems (MAS) have become increasingly relevant for developing distributed and dynamic intelligent environments (Corchado et al. 2007). One of the advantages of the agents is their adaptability to work in mobile devices, so they support wireless communication (Wi-Fi, Bluetooth,WiMAX, UMTS, etc.) which facilitates the portability to a wide range of mobile devices. This advantage makes the agents and multiagent systems appropriate to be applied to the development of ubiquitous and mobile environments and to obtain context information. Agents are computational entities that can be characterized through their capacities in areas such as autonomy, reactivity, pro-activity, social abilities, reasoning, learning and mobility (Wooldridge and Jennings 1995). These capacities make the multi-agent systems appropriate for constructing intelligent environments. An agent can act as an interface between the user and the rest of the elements of the intelligent environment. Furthermore, given the adaptability of agents to mobile devices (with low memory and processing resources), it is possible to provide ubiquitous and transparent interactions, even personalizing the user access. If we can provide an agent with the appropriate reasoning mechanism, for example a CBR system, it could be able to adapt itself to environmental changes or make predictions based on previous knowledge or experience (Corchado and Laza 2003). In this sense an agent which integrates a CBR system will be context-sensitive and will take decisions allowing it to automatically adapt itself to the changes on its surroundings.

Certain interface agents incorporate intelligent mechanisms to interact with the users of the multiagent system. Moreover, an agent can integrate certain middleware that allows it to interact with different context sensitive technologies, such as radio frequency identification (RFID) (Sokymat 2006), automation devices (ZigBee) (ZigBee Standards Organization 2006), etc. This last agent type will be able to obtain information of its surroundings in execution time, as well as to respond to the environmental changes. Then, if a CBR-based intelligent agent could be able to exchange information with the agents specialized in obtaining context sensitive information, and interact with interface agents, it could be possible to construct intelligent environments allowing personalized services. Figure 2 shows a scheme of such CBRbased intelligent environment. In Figure 2 it is possible to observe how users can gain access to the system by means of their personal agent installed in mobile devices or personal computers. Besides, it is possible to observe how different technologies integrate within the multiagent system in order to achieve the objectives of the intelligent 


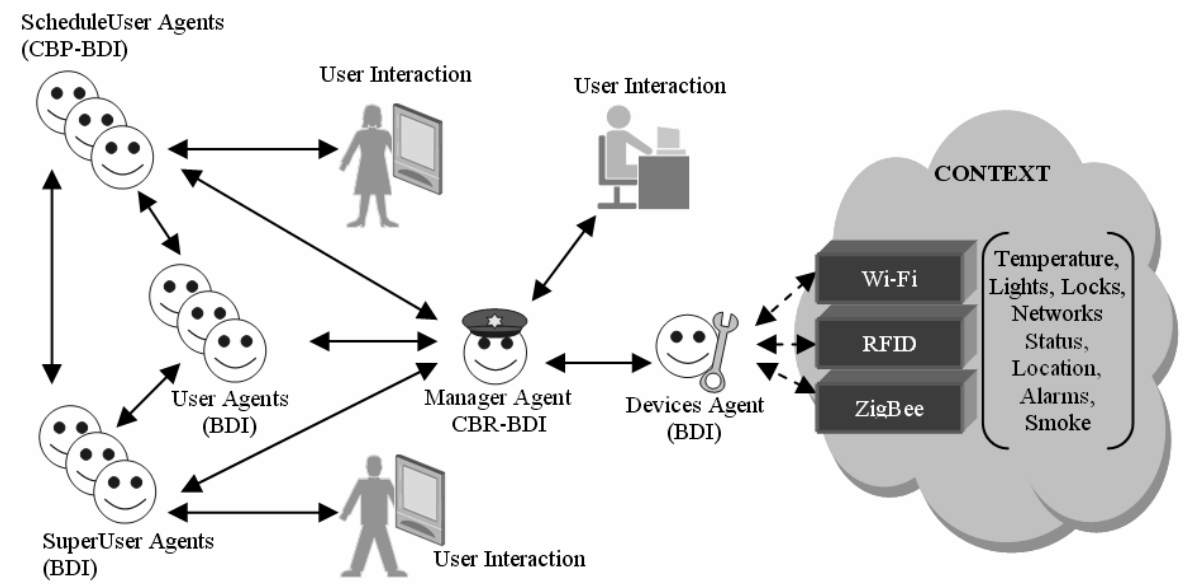

Fig. 2. Architecture scheme for intelligent environments construction

environment. The core of the system is the CBR agent, which provides reasoning and planning mechanisms.

\section{CBR Planning Mechanism}

There are many industrial environments requiring dynamic planning systems that allow them to respond to changes in the environment and to provide efficient plans in execution time, for example, optimising the working rotas. A CBP planning mechanism is a type of CBR system (Bajo et al. 2007) specialized on plan generation. The CBP presented within this work calculates the most re-plan-able intention (MRPI), which is the plan than can be easily substituted by others in case the initial plan gets interrupted. In a dynamic environment, it is important to have an alternative plan to maintain the efficiency of the system. The MRPI avoids coming back to the initial plan. It uses the concept of a geodesic plan: "plan of minimum risk" or the plan with a higher density function of plans around it. In an environment that changes unpredictably, any plan that is distal to the geodesic plan means that a certain risk is accepted. The CBP mechanism follows the 4 stages of a CBR system (Retrieval, Reuse, Review and Retain). During each reasoning stage the CBP performs the following actions:

Retrieval stage. During this step the CBP mechanism selects the plans from the plan-base, which are similar or contain similar information to the problem case that describes the aims of a given user and his/her restrictions and user profile. For the recovery of similar cases to the current problem, the multivariate technical of conglomerates analysis was used, that is the hierarchical method. The reason that the method of conglomerates analysis was chosen, is because the recovered cases will be more similar to the current problem than with other methods. Since it is an analysis type in which the variables are considered from a vectorial point and the comparisons are made keeping in mind the components of that vector. If there are two cases that have all the same components except one, this method no longer considers them the same. The general idea consists of distributing $\mathrm{n}$ data, of dimension $\mathrm{p}$, in "conglomerates" or 
groups (clusters) formed by data that are "similar to each other". To define the conglomerates, it is necessary to choose distances and measures of previous similarity. The usual distances are the euclidean and the distance of Mahalanobis. The algorithm of grouping hierarchical begins with $\mathrm{n}$ conglomerates (one for each individual observation). In successive steps the conglomerates unite for likeness. The process of the algorithm can be graphically represented in a dendrogram. The number of conglomerates can decide the starting from the dendrogram, stopping the process of mass at the moment that the application of the algorithm takes to unite conglomerates that are very distant (Martin 2001).

Reuse stage. During this stage the CBP mechanism creates a vectorial hyper dimensional space that includes all the problem restrictions (time, food, equipment, rooms, etc.) using a B-splines technique. Then all possible plans that satisfy a given user requirement are identified. Such plans are the geodesic curves (of the vectorial hyper dimensional space), which can be calculated using the Euler Theorem (Bellman 1957). Such geodesic plans guaranty minimum risk and constant efficiency, while satisfying the user objectives. The geodesic plan with the Minimum Jacobi Field will be the most re-plan-able intention. The Minimum Jacobi Field identifies the plan with more density of the other plans around it, and therefore, if the plan gets interrupted, a closer plan will be easily identified to carry out the user objectives. The CBP system recommends their users to use the most re-plan-able intentions.

Review stage: During the revision stage the user confirms the plan, indicating his/her satisfaction degree.

Retain stage: During the retain stage the plans are stored depending on their efficiency.

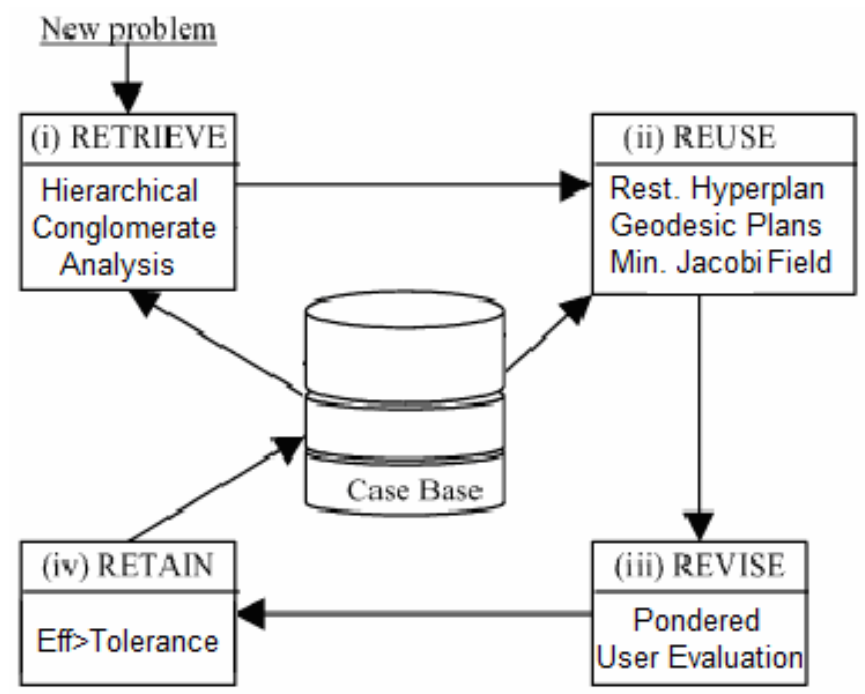

Fig. 3. Case-based planning mechanism for dynamic environments 
In Figure 3 it is possible to observe the steps carried out in each of the stages of the CBP system. The reuse stage implements the MRPI replaning mechanism (Bajo et al. 2007). When an interruption occurs, the system initiates a new CBP cycle, taking into account the tasks previously accomplished. That is, in the new retrieval stage, plans with a similar problem description to the current situation (after the interruption) will be recovered. The MRPI guarantees that at least some of the plans closest to the initial geodesic plan will be recovered (the rest of the plans are not valid anymore because of the restrictions, that the tasks have already accomplished, etc.) together with new plans. The proposed CBP mechanism has been tested in a real environment as shown in the next section.

\section{Case Study: Geriatric Residences}

The CBP planning mechanism can be very useful to facilitate planning or scheduling tasks in dynamic industrial environments. In this work we have focused on geriatric residences because they are one of the objectives of Ambient Intelligence (Friedewald and Da Costa 2003). The system prototype can easily be implemented on diverse dynamic scenarios, with some changes according to the users and project necessities. The CBP presented in this work focuses on scheduling the nurses working day and takes into account nurses' profiles, tasks that must be completed, available time and resources. The CBP needs to interact with its surroundings: patients, nurses, doctors, management personnel and intelligent devices.

\subsection{System Overview}

A real scenario that has been studied, the Alzheimer Santísima Trinidad Residence of Salamanca, has collaborated in the development of the intelligence environment presented here, providing their know-how and experimenting with the prototype developed. This residence is intended for people over 65 years, and has the following services and facilities available among others: TV room, geriatric bathroom, hairdressing salon, medical service, religious attention, occupational therapy, technical assistance, terrace, garden, laundry service, clothes adjustment, infirmary, reading room, living room, visitor room, cafeteria, social worker, chapel, elevator, customized diet, and multipurpose room. The residence has been equipped with RFID and Wi-Fi technologies as can be seen in Figure 4. 42 ID door readers (Hitag HT RM401and mobile WorkAbout Pro RFID) have been installed, one on each door and elevator, 4 controllers, one at each exit, one on the first floor hall and another on the second floor hall, and 36 bracelets (Sokymat ID Band Unique Q5 with a chip Hitag S 256) (Sokymat 2006), one for each patient and the nurses, shown in Figure 4. The ID door readers get the ID number from the bracelets and send the data to the controllers which send a notification to the Manager agent.

The intelligence environment has been modelled by means of four different types of agent, as can be seen in Figure 4:

- Patient agent manages the patient's personal data and behaviour (monitoring, location, daily tasks, and anomalies). Every hour it validates the patient's location, 
monitors the patient's state and sends a copy of its memory base (patient's state, goals and plans) to the manager agent in order to maintain backups. The patient's state is instantiated at execution time as a set of beliefs and these beliefs are controlled through goals that must be achieved or maintained. The beliefs that were seen to define a general patient's state at the Santísima Trinidad Residence of Salamanca were: weight, temperature, blood pressure, feeding (diet characteristics and next time to eat), oral medication, parental medication, posture change, toilet use, personal hygiene, and exercise. The beliefs and goals used for every patient depend on the plan (treatment) or plans that the doctors prescribe. The patient's agent monitors the patient's state by means of the goals. To know if a goal has been achieved or has failed, it is necessary to maintain continuous communication with the rest of the agents. At least once per day, depending on the corresponding treatment, the patient agent must contact the nurse agent. The patient's agent must have periodic communication with the doctor agent. Finally the patient agent must ensure that all the actions indicated in the treatment are fulfilled.

- The manager agent plays two roles, the security role that controls the patients' location and manages locks and alarms; and the Manager role that manages the medical record database and the doctor-patient and nurse-patient assignment. It must provide security for the patients and medical staff and the patients, doctors and nurse assignment must be efficient. The Manager agent controls all the tasks that must be accomplished in the residence. These tasks are assigned to the Nurse and Doctor agents. The Manager agent integrates a CBR mechanism to assign tasks to each nurse or doctor. The agent uses the experience obtained in previous assignments together with the nurses and doctor profiles and the time restrictions in order to optimize the tasks distribution.

- The doctor agent treats patients. The Doctor agent needs to interact with the Patient agent to order a treatment and receive periodic reports, with the Manager agent to consult medical records and assigned patients, and with the Nurse agent to ascertain the patient evolution.

- The nurse agent schedules the nurse's working day obtaining dynamic plans depending on the tasks needed for each assigned patient. Nurse agent manages nurses' profiles, tasks, available, time and resources. The generated plans must guarantee that all the patients assigned to the nurse are given care. The nurse can't exceed 8 working hours. Every agent generates personalized plans depending on the nurse's profile and working habits. The Nurse agent integrates a CBP planning mechanism to schedule the nurse's working day. Every day the Nurse agent receives the tasks assigned by the Manager agent. The Nurse agent uses the CBP mechanism to schedule the tasks and provides the nurse with a plan. Moreover, if any kind of interruption occurs during the plan execution, the Nurse agent uses its replanning capability to resolve the problem.

Manager and Patient agents run in a central computer, but the Nurse agents run on mobile devices, so a robust wireless network has been installed as an extension to the existing wired LAN. 


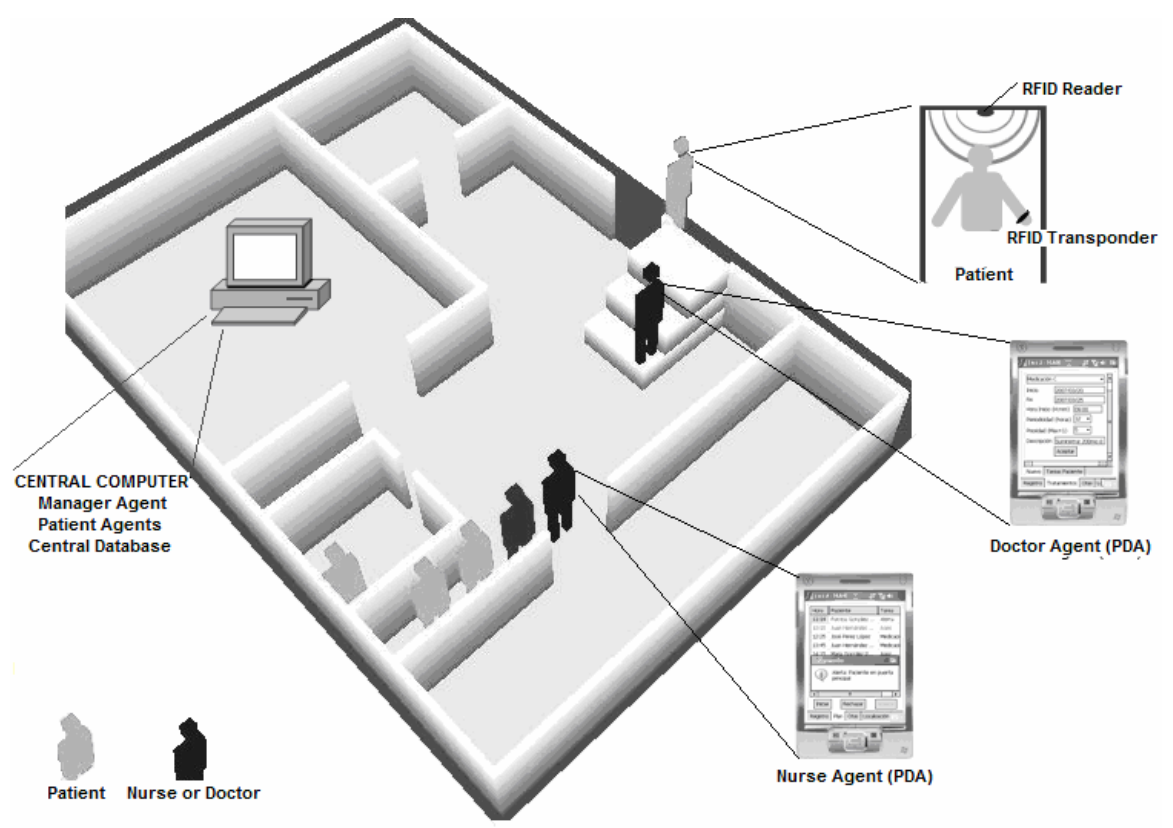

Fig. 4. Wireless technology organization schema

\subsection{Embedding a CBR System within a Deliberative Agent}

The CBR planning mechanism presented in Section 3 has been used to model the scheduling of the nurses working times dynamically in a geriatric residence (Corchado et al. 2007). A large quantity of measurements have been taken in order to standardise the time taken to arrive at a given room, or to take a patient from one room to another (depending on the level of dependence of the patient). These times are included directly in the time assigned for each task. The CBP Nurse agent constructs plans as a sequence of tasks that need to be carried out by a nurse.

The first step when working with CBRs is obtaining a definition of the case structure. A case structure is composed of three elements: a problem description, the solution applied to solve the problem and the result obtained for the solutions applied (Aamodt and Plaza 1994). For the current problem the case is described as follows:

- Problem description: Describes the initial information available for generating a plan. As can be seen in Table 1 the problem description for planning a nurse's working day consists of a case identification, the list of tasks that the nurse has to accomplish, the nurse's profile, the nurse's preferences and temporal restrictions.

Where the structure of a Task can be observed in Table 6 , and the structure corresponding to a nurse profile can be observed in Table 5. 
Table 1. Problem description for a planning a nurse working day

\begin{tabular}{ll}
\hline Problem Description Field Field Type \\
\hline CaseId & Integer \\
Tasks & ArrayList of Tasks \\
Nurse Profile & NurseProfile \\
Nurse Preferences & NursePreferences \\
Restrictions & ArrayList of Restriction \\
\hline
\end{tabular}

Solution: Describes the actions carried out in order to solve the problem description. As can be seen in Table 2 it contains the case identification, the timetables and the priorities for each task and the route or sequence of tasks.

Table 2. Solution (Plan) proposed to the nurse

\begin{tabular}{ll}
\hline Solution Field & Field Type \\
\hline Case d & Integer \\
TimeTables & ArrayList of TimeTable \\
Route & Route \\
\hline
\end{tabular}

Where the TimeTables field contains a list of Timetable elements. A Timetable element, as shown in Table 3, indicates the identification of a task, the priority of a task, the desired starting and finishing times for a task and the room identification if required.

Table 3. Timetable for a task

\begin{tabular}{ll}
\hline TimeTable & Field Type \\
\hline Priority & Float \\
StartTime & Time \\
FinishTime & Time \\
Task & Float \\
Room & Float \\
\hline
\end{tabular}

The Route field contains a description of the activities carried out in a room of the residence, as shown in Table 4.

Table 4. Route describing the activities executed by a nurse in a given room

\begin{tabular}{ll}
\hline Route Fields & Field Type \\
\hline room & Room \\
ArrivalTime & Time \\
ServiceTime & Time \\
patient & Patient \\
Tasks & ArrayList of Task \\
NextRoom & Route \\
\hline
\end{tabular}


- Efficiency: contains the case id and a number indicating the plan efficiency

For each of the rooms in the geriatric residence, the system manages information related to the room location (in coordinates), access (doors) location (in coordinates), room type and resources available.

In our case study, at the beginning of each working day, every nurse receives a list of tasks that must be completed. The tasks must be assigned to the nurses. It is necessary to take into account that each nurse has a different profile according to their qualification and the tasks that they usually carry out. It is considered appropriate to manage the profiles of the nurses because there are some nurses who perform tasks with greater skill or who carry out tasks faster. The structure of a nurse profile can be observed in Table 5. Table 5 shows the abilities of a nurse, her service time and timetable preferences and restrictions.

Table 5. NurseProfile

\begin{tabular}{ll}
\hline NurseProfile Fields & Field Type \\
\hline Skills & ArrayList of Skill \\
ServiceTime & Time \\
Timetable preferences & Date \\
TImetable restrictions & Date \\
\hline
\end{tabular}

One of the Manager agent's duties is the assignation of nurses to patients. This assignation is carried out through a CBR reasoning motor integrates within the Manager Agent. When a new assignation of tasks needs to be carried out to the nurses, both past experiences, such as the nurse profile, and the needs of the current situation are retrieved. In this way tasks are allocated to a nurse. These tasks may correspond to the same patient or to a number of patients. The task structure is shown in Table 6 . Moreover, as mentioned above, the profile of each nurse is taken into account. For example, not all nurses are equally qualified for rehabilitation. If one nurse is more qualified in the area, she will be allocated the patients whose need for rehabilitation are greater, always taking into account that the nurse cannot work more than 8 hours, so that the number of patients assigned depends on the time needed to carry out the rehabilitation. The Manager agent takes into account how those patients who receive rehabilitation are improving, the arrival of new patients, holiday rotas etc. As such, the allocation of tasks needs to be set on a daily basis.

Table 6. Task structure

\begin{tabular}{ll}
\hline Task Field & Field Type \\
\hline taskData & TaskData \\
ServiceTime & Time \\
Date & Date \\
Room & Room \\
Resources & ArrayList of Resource \\
\hline
\end{tabular}


Once the assignation of tasks to a nurse has been completed, the assignation is communicated to the corresponding Nurse agent. That is, the Nurse agent receives a new problem description needing to be resolved. From this moment on, the CBP planning process begins. The Nurse agent must take into account the time that the nurse has available and the time required for each task. Moreover, the resources available and the location of the patients involved are also taken into account. Then the stages of the CBP cycle are executed:

- Retrieval: In the retrieval stage, the descriptions of similar problems are recovered. In order to do this, the Nurse agent allows the application of the hierarchical conglomerate analysis algorithm (Martin 2001). In this step, those problem descriptions found within a range of similarity close to the original problem description are recovered from the cases base. Every retrieved case "k" related to a task "i", is represented by means of a vector $X_{k i}$.

$$
X_{k i}=\left(x_{k i 1}, x_{k i 2}, x_{k i 3}, x_{k i 4}, x_{k i 5}, x_{k i 6}, x_{k i 7}, x_{k i 8}, x_{k i 9}\right)
$$

Where $x_{k i 1}$ indicates the priority for task i, $x_{k i 2}$ indicates the patient location, $x_{k i 3}$ indicates the start time, $x_{k i 4}$ indicates the finish time, $x_{k i 5}$ indicates the resources required, $x_{k i 6}$ indicates the service rate, $x_{k i 7}$ indicates the nurse assigned, $x_{k i 8}$ indicates the plan executed and $x_{k i 8}$ indicates the nurse qualification.

Every task "i" corresponding to the new problem " $\mathrm{j}$ " is represented by jeans of the vector $Y_{j i}$

$$
Y_{j i}=\left(x_{j i 1}, x_{j i 2}, x_{j i 3}, x_{j i 4}, x_{j i 5}, x_{j i 6}\right)
$$

Where $x_{j i 1}$ indicates the priority for task i, $x_{j i 2}$ indicates the patient location, $x_{j i 3}$ indicates de start time for task $\mathrm{i}, x_{j i 4}$ the finish time for task $\mathrm{i}$, $x_{j i 5}$ the resources required to accomplish the task and $x_{j i 6}$ the nurse qualification required.

In order to find those cases containing the task "i" which are similar to the current problem, the euclidean distance is applied. The first five vector components of each case are significant, so they are taken into account. In the first step, the closest case to the current problem is grouped. In the next step, those cases grouped in the first step are considered as a new group, the distances (similarities) among groups are recalculated for the new configuration and the two closest groups are put together. This process is repeated successively until only one group is obtained. The final result is a dendogram, a typical graph used to represent clustering analysis.

Reuse: Once the most similar problem descriptions have been recovered, the reuse stage recovers the solutions (plans) associated with them. One solution contains all the plans (sequences of tasks) that were carried out in order to achieve the objectives of the Nurse agent for a problem description (assuming that replanning is possible) in the past, as well as the efficiency of the 
solution being supplied. The recovered solutions are combined, as explained in (Corchado et al. 2007), to construct a plan. At this time the Nurse agent takes control of the processing of the plan (scheduling). The re-planning mechanism is centred around the objectives and resources needed by each task, as well as on the objectives that the nurse needs to perform and the resources available in order to carry out the global plan. The objectives or global plans that each nurse has, are to attend to the patients and not to work for over eight hours. The time available is a problem restriction. This available time will influence the hyper plan of restrictions, specifically, the range of positive values that the $\mathrm{z}$ axis takes from this hyper plan. The resources necessary for some of the tasks are food, equipment and rooms. Finally, the Nurse agent takes care of incidents and interruptions that may occur during replanning.

A plan can be interrupted for different reasons. Those which have been taken into account within the residence are: a resource failure, a patient suffers some sort of crisis and requires unforeseen attention, the patient has an unexpected visit or that visits to the patient have exceeded the permitted time or an emergency situation. If the planner finds itself in a situation where the plan is interrupted, it rejects the initial plan and seeks an alternative one. The first thing that needs to change is the task order, attempting to maintain the assignation originally allocated by the Manager agent. The new plan must meet the initial objectives. In the event that this is impossible, the nurses will need to be reassigned. This reassignment will attempt to limit changes to a minimum. For reassignment it is necessary to take into account the tasks that were assigned to the nurses, the development of the plans (which tasks have been carried out and which still need to be done) and the profiles of the nurses (prioritising preparation for the task that cannot be covered). The nurse who is assigned the task should replan in order to include the new task. In the event that the replanning is positive (the tasks that still need to be done and the new task can be carried out) the process is complete. If the replanning is negative, the next nurse down in the ranking will be used. - Review: The nurse evaluates the plan

- Retain: Lastly, depending on the efficiency of the plan, it will be stored together with its level of efficiency within the beliefs base.

\section{Results and Conclusions}

The CBP planning mechanism which allows replanning in execution time, integrated within an intelligent agent, has been tested over the last few months. To test the system 30 patient agents, 10 nurse agents, 2 doctor agents and 1 manager agent were instantiated. During the testing period the systems usefulness has been evaluated from different points of view. The system, which in turn improves patient care, facilitates the more flexible assignation of the working shifts at the residence; since the workers have reduced the time spent on routine tasks and can assign this time to extra activities, such as exercising the patients, learning, carrying out leisure activities or just talking with the patients or with their families. Their work is automatically monitored, 
as well as the patients' activities. The stored information may be analysed with knowledge discovery techniques and may help to improve the quality of life for the patients and the efficiency of the centre. The security of the centre has also been improved in three ways: the system monitors the patients and guarantees that each one of them is in the right place; secondly, only authorised personnel can gain access to the residence protected areas, and thirdly, the information is stored in a more secure way using redundancy and generating continuous backups. The access to information has been protected in order to guarantee confidentiality.

The agent technology provides a framework for distributed problem solving and data communication. The characteristics of agents, such as autonomy, reactivity, proactivity, social abilities, reasoning, learning and mobility (Wooldridge and Jennings 1995) are adequate to fulfil the AmI necessities. Moreover, the incorporation of casebased reasoning mechanisms (Corchado and Laza 2003; Bajo et al. 2007) facilitates ubiquitous computation capabilities. An agent can act as an interface between the user and the environment. In this way, it is possible to get that the services provided by the multiagent system and the technology in the environment can be adapted to the user necessities. Finally, agents can be executed on mobile devices, which facilitate ubiquitous capabilities. These characteristics make the proposed architecture appropriate for developing AmI (Friedewald and Da Costa 2003) scenarios. The manager agent assigns tasks and monitors the patients and nurses locations. Nurse and Doctor agents run on handheld devices and provide easy-to-use interfaces.

The planning mechanism has been evaluated taking into account the characteristics of a CBR system (Aamodt and Plaza 1994). In this sense, the parameters that influence the efficiency of each of the stages of the proposed CBP cycle have been studied in detail. In Figure 5 it is possible to see the interaction between the variables

\section{$\%$ Satisfaction-Number of Cases}

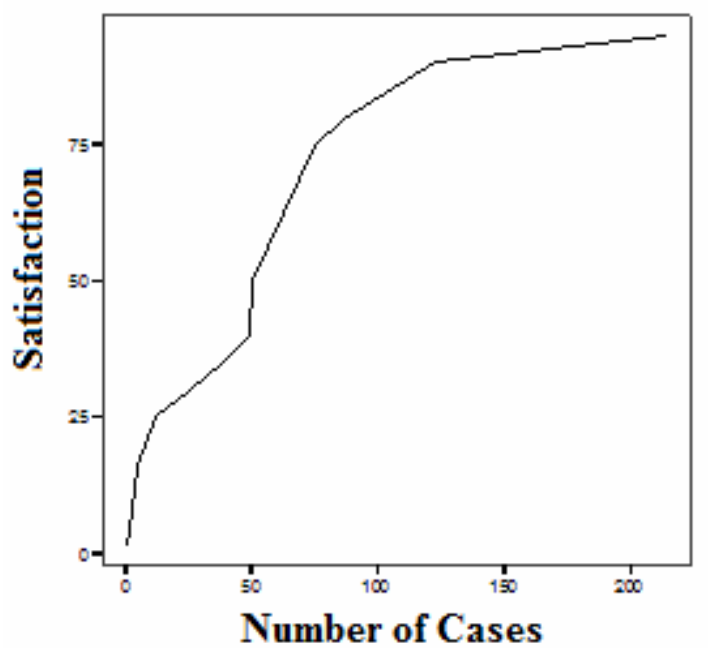

Fig. 5. \% Average satisfaction degree related to the average number of retrieved cases 


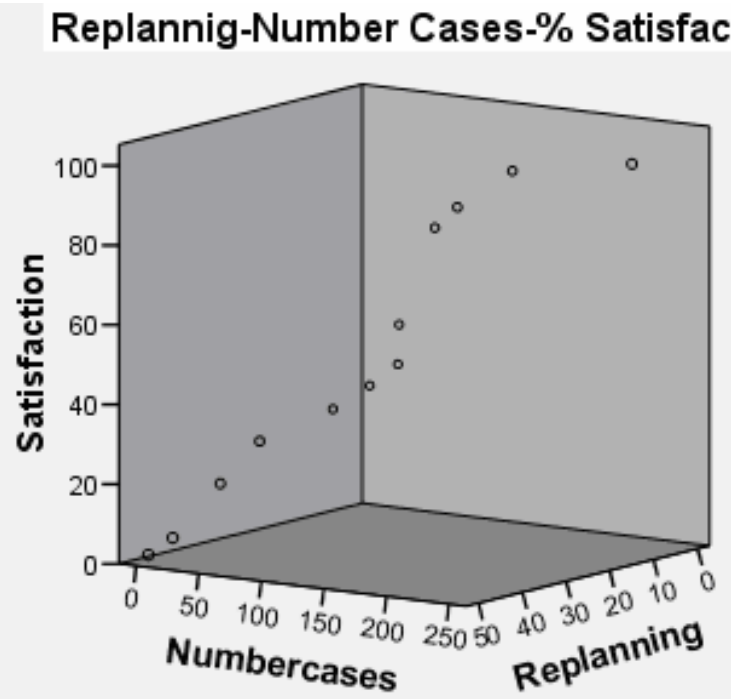

Fig. 6. Interaction between average number of cases retrieved, average number of replannings required per plan and the nurse satisfaction degree

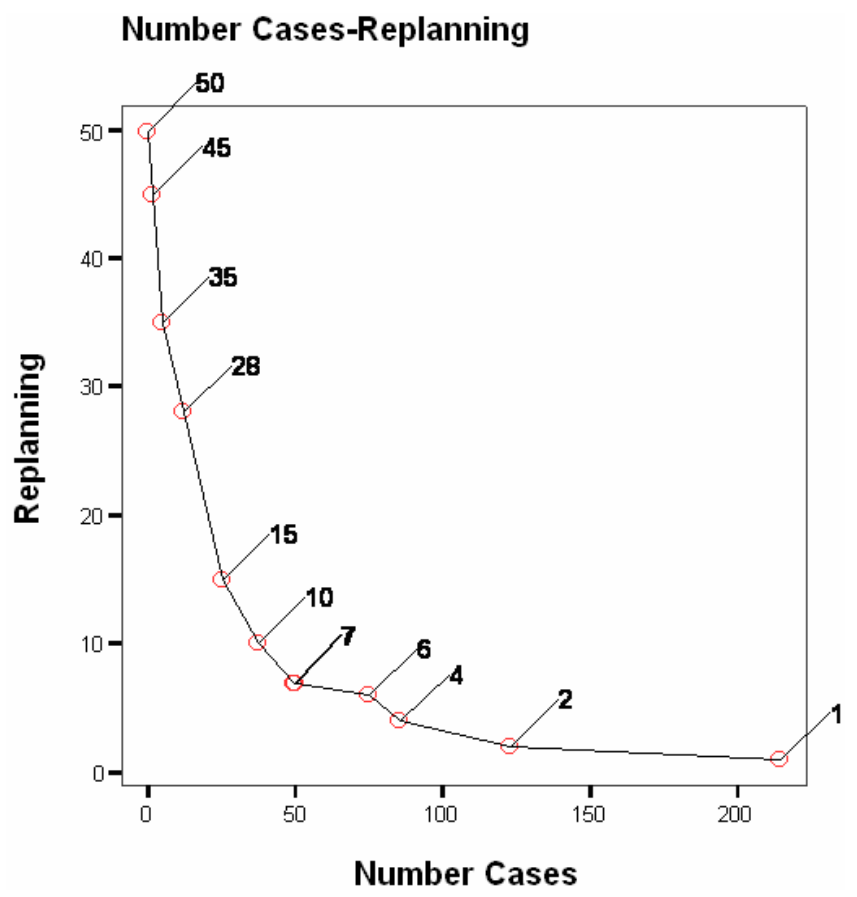

Fig. 7. Number of replannings related to cases retrieved 


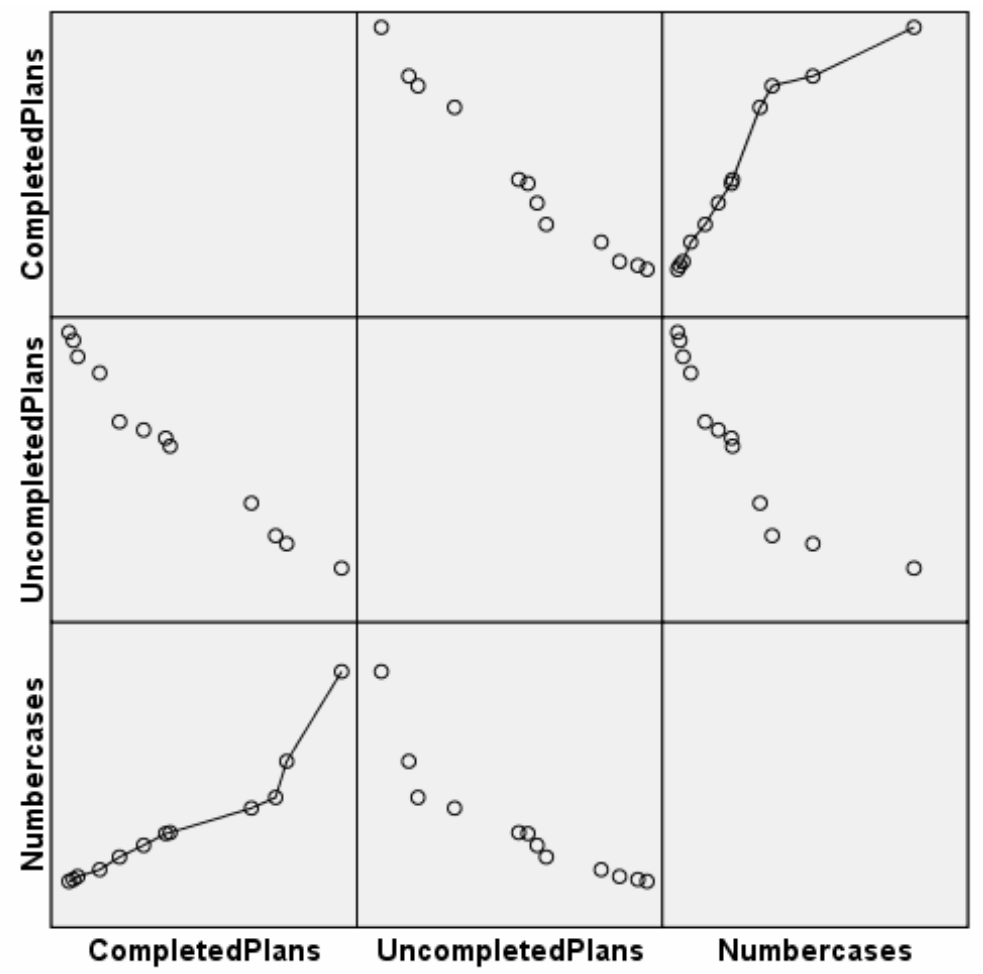

Fig. 8. Plans completed and plans failed related to the average number of cases retrieved

corresponding to the average satisfaction degree for a nurse related to the plan success and the number retrieved from the base case in order to provide the plan. As explained in Section 2, one of the key concepts in the efficiency of a CBR system consists of the retrieval algorithm implemented, and specifically, in the similitude metric applied. The success or efficiency of a plan can be calculated through the results obtained for each of the tasks of the plan and the evaluation provided by the nurse that has completed the plan. On the other hand, the number of retrieved cases allows evaluating the strategy proposed in the retrieval stage. The results presented in Figure 5 show how for a larger number of retrieved cases from the cases memory, the satisfaction degree indicated by nurses increases. That is, the nurses can better achieve their initial objectives when the number of similar past experiences available is larger. The relationship between the number of retrieved cases and the nurse satisfaction degree is stabilized for a number of cases around 110 and 200.

The case-based planning mechanism proposed in the frame of this research comes characterized by its capability for dynamic replanning in execution time. In order to obtain a better understanding of the success of the CBP planning system, it is of interest to take into account the average number or replanning actions executed for a plan. Figure 6 shows the interaction between the number of retrieved cases, the average satisfaction degree (percentage) and the average number of replans per plan in the proposed CBP system. In Figure 6 it can be observed how the number of retrieved 
cases increases, the satisfaction degree grows and the average number of replannings required per plan decreases.

In Figure 7 it has been represented in the interaction that exists between the average number of cases retrieved in order to solve a plan and the average number of replannings required. It is possible to detect an inverse relationship between both variables, that is, when the average number of retrieved cases increases the average number of replannings required decrease and vice versa. Moreover, the graph presents an inflection point of 50 retrieved cases, corresponding to an average of 7 replannings. This fact let's us conclude that from 50 retrieved cases on, the number of replannings decreases smoothly. It is necessary to notice that a CBR system needs initial knowledge and a certain learning period to provide good results.

Another good indicator to evaluate the CBP is the success of the plans suggested. The plans recommended to the nurses can be successfully completed (replanning can be necessary or not) or failed. In the same way as the average number of replannings and the nurse satisfaction degree was influenced by the number of cases retrieved. The number of plans successfully completed depends on the retrieval strategy implemented. Figure 8 presents a $3 \times 3$ matrix representing the interaction between the

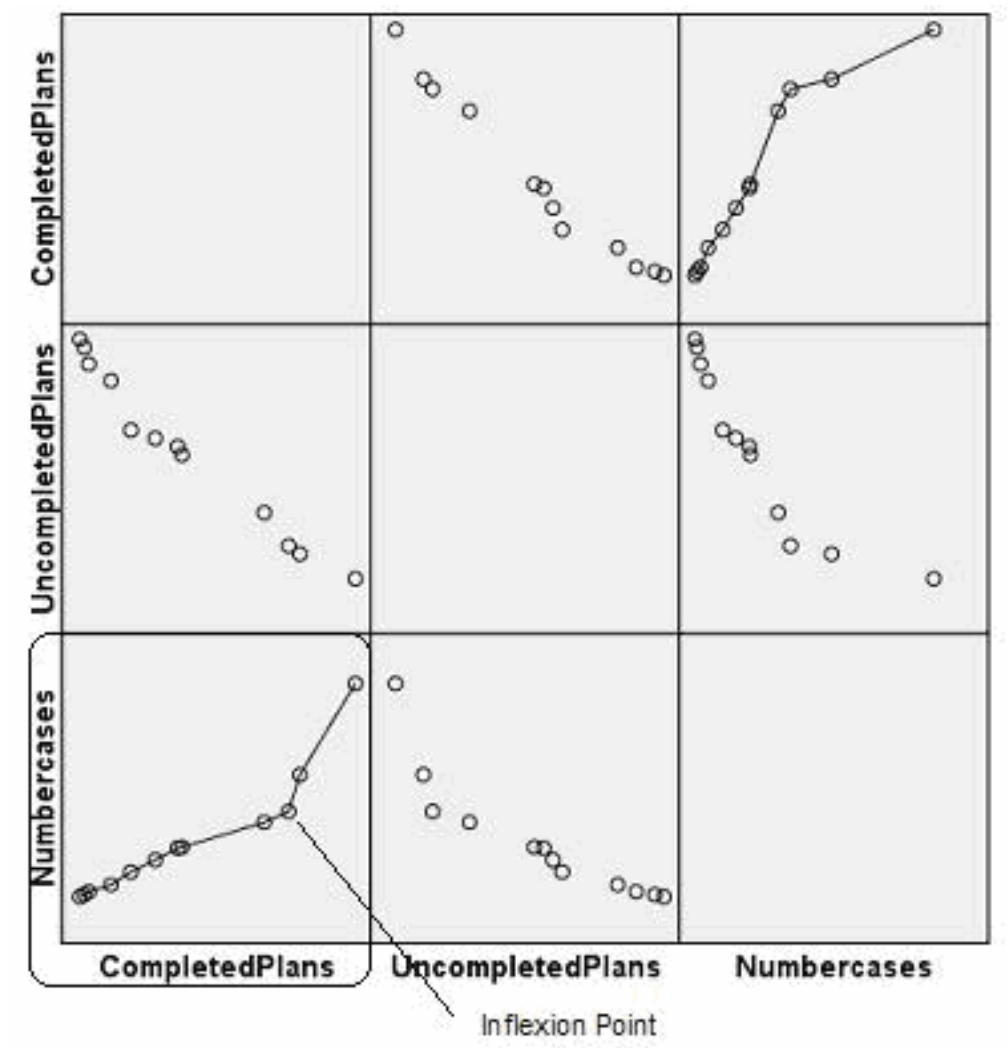

Fig. 9. Inflection point in the interaction between the average number of completed plans and the average number of retrieved cases 


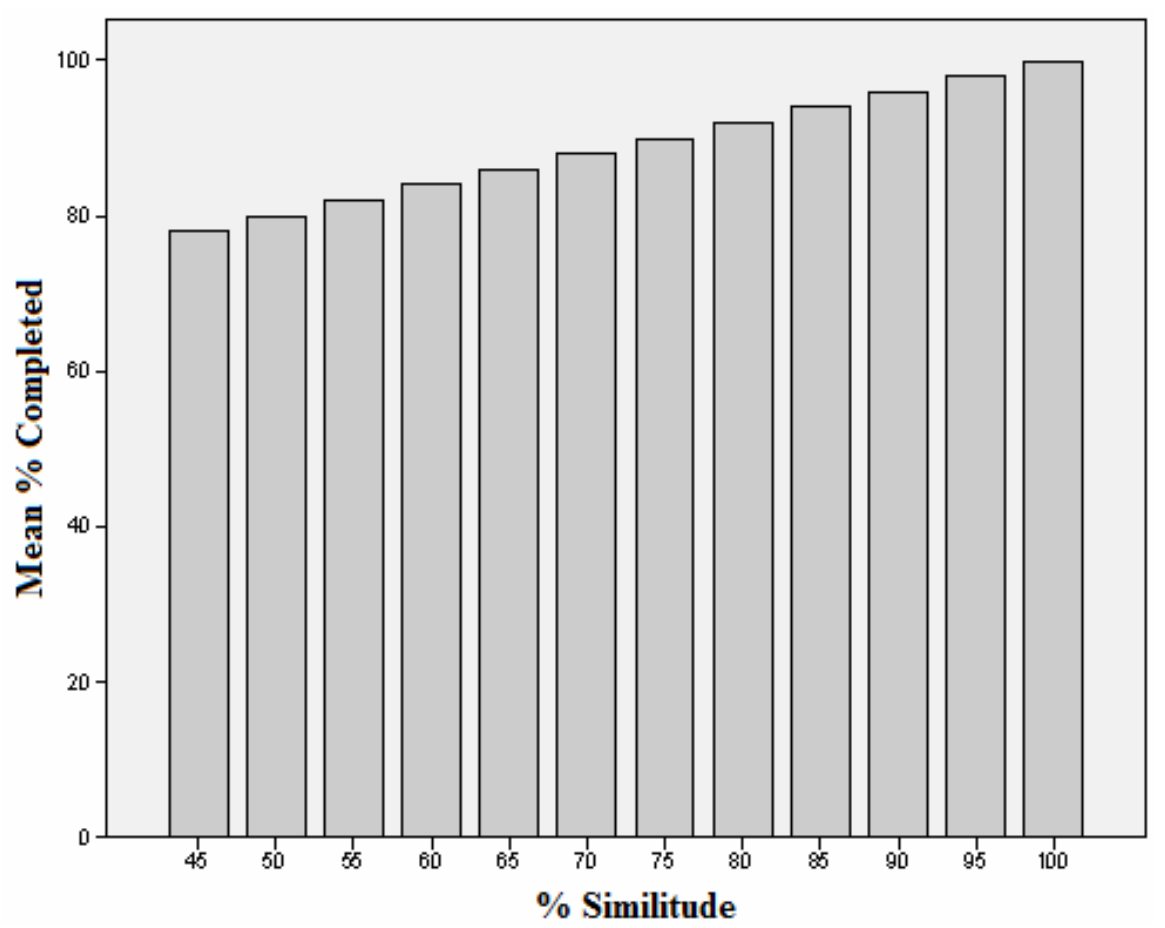

Fig. 10. $\%$ Plans completed related to the $\%$ of similitude for retrieved cases

average number of retrieved cases, the average number of completed plans and the average number of failed plans. Figure 8 let's us conclude that as the number of cases retrieved increases, the number of plans completed successfully increases and the number of plans failed decreases. In Figure 9 we can see that exits an inflection point in the row indicating the average number of retrieved cases and the column indicating the average number of plans completed. Such a point indicates, that from a certain number of retrieved cases on, the number of plans successfully completed stabilizes. Even, if the number of retrieved cases increases dramatically, the success for the proposed plans decreases.

Finally, the influence of the similitude degree obtained for the retrieved cases has been evaluated. Figure 10 presents the interaction between the percentage of plans successfully completed and the similitude degree obtained during the retrieval stage. In Figure 10 it can be seen that, generally, as more similar the retrieved cases are to the current problem, the possibility of plan success increases. CBP is based on the use of past experiences to resolve new problems. Obviously, if we can obtain past plans containing similar tasks and these plans were executed by nurses with similar skills, the probability of success increases. However, the CBP is highly affected by environmental changes, and the success for a plan depends not only on previous experiences but also on the incidents that can happen during the execution of the plan, which cause events of interruption and the corresponding replanning actions. 
In the future, several industrial environments will require the use of new technologies that allow the personnel to carry out their tasks more efficiently. We have shown the potential of the CBP planning mechanism in a distributed intelligent environment on health care, providing a way to respond to some challenges of health care, related for example to the identification, control and health care planning. In addition, the use of RFID technology on people provides a high level of interaction among users and patients through the system and is fundamental in the construction of the intelligent environment. Furthermore, the use of mobile devices, when used correctly, can facilitate social interactions and knowledge transfer. The work presented can be easily adapted to similar environments by configuring certain parameters of the planning mechanism.

\section{Acknowledgements}

This work has been partially supported by the MCYT projects TIN2006-14630-C0303/ and TIC2003-07369-C02-02 and the JCYL project SA104A05. Special thanks to Sokymat by the RFID technology provided and to Telefónica Móviles (Movistar) for the wireless devices donated.

\section{References}

Aamodt, A., Plaza, E.: Case-Based Reasoning: Foundational Issues, Methodological Variations, and System Approaches. AI Communications 7, 9-59 (1994)

Bajo, J., Corchado, J.M., Rodríguez, S.: Intelligent Guidance and Suggestions Using CaseBased Planning. In: Weber, R.O., Richter, M.M. (eds.) ICCBR 2007. LNCS (LNAI), vol. 4626, pp. 389-403. Springer, Germany (2007)

Bratman, M.E.: Intentions, Plans and Practical Reason. Harvard University Press, Cambridge, M.A. (1987)

Bellman, R.E.: Dynamic Programming. Princeton University Press, Princeton, New Jersey (1957)

Camarinha-Matos, L., Afsarmanesh, H.: Design of a Virtual Community Infrastructure for Elderly Care. In: Camarinha-Matos, L. (ed.) Proceedings of PRO-VE 2002, Sesimbra, Portugal (2002)

Corchado, J.M., Laza, R.: Constructing Deliberative Agents with Case-based Reasoning Technology. International Journal of Intelligent Systems 18(12), 1227-1241 (2003)

Corchado, J.M., et al.: Intelligent Environment for Monitoring Alzheimer Patients. In: Agent Technology for Health Care. Decision Support Systems, Elsevier Science, Amsterdam (2007)

Emiliani, P.L., Stephanidis, C.: Universal access to ambient intelligence environments: opportunities and challenges for people with disabilities. IBM Systems Journal (2005)

Foster, D., McGregor, C., El-Masri, S.: A Survey of Agent-Based Intelligent Decision Support Systems to Support Clinical Management and Research. In: Armano, G., et al. (eds.) Proceedings of MAS*BIOMED 2005, Utretch, Netherlands (2005)

Friedewald, M., Da Costa, O.: Science and Technology Roadmapping: Ambient Intelligence in Everyday Life (AmI@Life). Working Paper. Institute for Prospective Technology Studies IPTS, Seville (2003) 
Kohn, L.T., Corrigan, J.M.: Donaldson, To Err is human: Building a Safer Health System. Committee on Quality of Health Care in America, Institute of Medicine. National Academy Press, Washington, DC (1999)

Martín, Q.: Course about treatment of statistics data with SPSS, Hesperides (2001)

Nealon, J., Moreno, A.: Applications of Software Agent Technology in the Health Care domain. Whitestein series in Software Agent Technologies, Birkhauser (2003)

Sokymat (2006), http://www.sokymat.com

Susperregi, L., et al.: Una arquitectura multiagente para un Laboratorio de Inteligencia Ambiental en Fabricación. 1er. Taller de Desarrollo de Sistemas Multiagente (DESMA). Málaga, Spain (2004)

Wooldridge, M., Jennings, N.R.: Agent Theories, Architectures, and Languages: A Survey. In: Wooldridge, Jennings (eds.) Intelligent Agents, pp. 1-22. Springer, Heidelberg (1995)

ZigBee Standards Organization, ZigBee Specification Document 053474r13. ZigBee Alliance (2006) 